\title{
Revealing potential immunotherapy targets through analysis of a ceRNA network in human colon adenocarcinoma
}

\author{
Changhao Li, Zhenyu Zhu, Qingsheng Hou, Bishi Wang, Lei Zou, Luguang Liu, Weipeng Gong, \\ Hongliang Guo
}

Department of Gastrointestinal Surgery, Shandong Cancer Hospital and Institute, Shandong First Medical University and Shandong Academy of Medical Sciences, Jinan, China

Contributions: (I) Conception and design: C Li; (II) Administrative support: W Gong, H Guo; (III) Provision of study materials or patients: C Li; (IV) Collection and assembly of data: All authors; (V) Data analysis and interpretation: C Li; (VI) Manuscript writing: All authors; (VII) Final approval of manuscript: All authors.

Correspondence to: Dr. Weipeng Gong; Prof. Hongliang Guo. Shandong Cancer Hospital and Institute, Shandong First Medical University and Shandong Academy of Medical Sciences, Jinan 250001, China. Email: cong2008cong@163.com; hlguo2007@163.com.

Background: Microsatellite instability-high (MSI-H) is a special type of human colon adenocarcinoma (COAD) that responds well to immunotherapy. MicroRNAs (miRNAs) and long non-coding RNAs (lncRNAs), which are important members of competing endogenous RNAs (ceRNAs) networks, are involved in the tumorigenesis and development of MSI-H COAD. This study aimed to establish a ceRNA network for MSI in COAD to identify targets and prognostic markers that may explain the effects of immunotherapy. Methods: COAD sequencing data were extracted from The Cancer Genome Atlas (TCGA), after which differentially expressed miRNAs, lncRNAs, and mRNAs were determined according to microsatellite status. After building a network based on the ceRNA hypothesis, the relationships between microsatellite status and clinical features were explored. Biological processes in the Gene Ontology (GO) and the Kyoto Encyclopedia of Genes and Genomes (KEGG) databases were analyzed for specific miRNAs, lncRNAs, and mRNAs. Survival analysis was used to identify potential biomarkers.

Results: Based on the inclusion criteria, a total of 363 COAD samples were obtained from TCGA. Strict screening criteria were used to identify differentially expressed RNAs in the MSI-H and microsatellite-stable groups, with 82 miRNAs, 1,280 lncRNAs, and 2121 mRNAs obtained (fold change $>2$, false discovery rate $<0.01$ ). Based on the RNA interaction mechanism, a miRNA-lncRNA-mRNA network was constructed, through which a subnetwork composed of 5 miRNAs was discovered. hsa-miR-31-5p, hsa-miR-302a-3p, hsamiR-302b-3p, hsa-miR-302d-3p, hsa-miR-3619-5p and the RNAs interaction with them have the potential to become novel targets to change the effect of existing immunotherapy. GO and KEGG analyses showed that these differentially expressed miRNAs, lncRNAs, and mRNAs may play key roles in tumorigenesis, tumor development, and drug efficacy, with natural killer cells potentially becoming the next emerging targets for immunotherapy enhancement. Moreover, survival analysis identified 10 lncRNAs as potential survival markers.

Conclusions: This study identified novel immunotherapy targets and revealed potential biomarkers for COAD according to microsatellite status.

Keywords: Colon adenocarcinoma (COAD); natural killer cells; ceRNA network; biomarkers; bioinformatics analysis

Submitted Oct 08, 2021. Accepted for publication Nov 23, 2021.

doi: $10.21037 /$ tcr-21-2380

View this article at: https://dx.doi.org/10.21037/tcr-21-2380 


\section{Introduction}

Estimates from 2018 showed that colorectal cancer (CRC) accounted for $6.1 \%$ of the more than 1 million new cancers worldwide, with a mortality rate of $9.2 \%$, making it the fourth most morbid and second most fatal cancer (1). Comprehensive treatment strategies based on staging, including preoperative adjuvant radiotherapy and chemotherapy, surgical treatment, postoperative systemic therapy, and targeted therapy, are still the mainstream approaches for treating CRC (2-4). Over the past decade, immunotherapy has been increasingly used for the treatment of CRC due to the promising effects of immune checkpoint inhibitors (ICIs) on various solid tumors which were previously difficult to treat. The phase III KEYNOTE-177 trial showed that pembrolizumab exhibited greater efficacy for microsatellite instability-high (MSI-H)/mismatch repair-deficient (dMMR) metastatic CRC compared to chemotherapy, raising the median progression-free survival (PFS) from 8.2 to 16.5 months (5). Such findings prompted the Food and Drug Administration (FDA) to authorize pembrolizumab for first-line immunotherapy of patients with MSI-H/dMMR metastatic CRC, with MSI-H/ $\mathrm{dMMR}$ disease having been identified as a key biomarker for immunotherapy. Therefore, identifying the underlying mechanism of MSI-H/dMMR status in CRC is imperative.

Microsatellites are short tandem repeat DNA sequences widely found in eukaryotic genomes. Most of them are located in the non-coding region of the gene or near the telomere region of the chromosome, probably due to mismatch repair-deficient (dMMR), which plays a pivotal role in maintaining genome stability. The length change in short repeats due to insertion or deletion is termed microsatellite instability (MSI) (6). CRC can be divided into 3 separate groups based on mutation patterns: mismatch repair-deficient or microsatellite instability-high (dMMR/ MSI-H), mismatch repair-proficient or microsatellite instability-low (pMMR/MSI-L), and microsatellite-stable (MSS) disease. Given that MSI-L CRC and MSS do not benefit from ICI treatment (7), there is an urgent need to determine how to sensitize these groups to immunotherapy.

Salmena et al. proposed the competitive endogenous RNA (ceRNA) hypothesis, which suggests that mRNAs, IncRNAs, and other non-coding RNAs can be used as natural miRNA "sponges" that participate in the process of RNA degradation and post-translational modification together with ordinary miRNA response elements (MREs) (8). Previous studies have confirmed that non-coding RNAs are involved in tumorigenesis, invasion, metastasis, and other biological processes involved in colon cancer (9). We need more research to discover whether MIRNA plays an important role in the formation of MSI-H CRC.

Colon adenocarcinoma (COAD) is an important subtype of CRC. Although previous studies have explored the ceRNA network in COAD metastasis (10), the connection between ceRNAs and microsatellite status has yet to be investigated in detail. Therefore, studies on the relationship between ceRNAs and microsatellite status may uncover the potential molecular mechanisms in the pathogenesis of MSI, as well as identify new biomarkers for predicting the effectiveness of immunotherapy. More importantly, we hope to find a possible way to enhance the effect of immunotherapy by analyzing the ceRNA network in different microsatellites status of COAD to cope with the current situation that MSS COAD and MSI-L COAD are not sensitive to immunotherapy and improve COAD patients' survival rate.

Given that The Cancer Genome Atlas (TCGA) database provides sequencing datasets of RNAs (including miRNAs) across a large number of COAD samples, we determined differentially expressed RNAs in COAD according to microsatellite status by analyzing available high-throughput RNA data in TCGA database. Subsequently, the Gene Ontology (GO) and Kyoto Encyclopedia of Genes and Genomes (KEGG) pathway enrichment databases were used to identify the pathways regulated by these differentially expressed RNAs, which may uncover potential mechanisms for the pathogenesis of MSI-H disease. We present the following article in accordance with the REMARK reporting checklist (available at https://dx.doi. org/10.21037/tcr-21-2380).

\section{Methods}

\section{Data acquisition}

The COAD RNA and miRNA sequencing data were downloaded from TCGA data portal (downloaded on September 28, 2020). Clinical information and microsatellite status information were downloaded from the Firebrowse database (http://www.firebrowse.org/) (downloaded on September 28, 2020). The inclusion criteria for COAD tissue samples were as follows: (I) samples with a pathologically confirmed diagnosis of COAD; (II) those with RNA-seq data, miRNA-seq data, and clinical information; (III) those corresponding to only one set of RNA sequencing data and 
miRNA sequencing data; (IV) those with microsatellite status information; (V) and those from the primary tumor. Accordingly, a total of 363 COAD samples were included in our analysis. The study was conducted in accordance with the Declaration of Helsinki (as revised in 2013).

\section{Analysis of differentially expressed RNAs according to microsatellite status}

Processing and analysis of sequencing data were performed using $\mathrm{R}$ (version 4.0.2). The sequencing data of 363 samples were divided into 3 groups according to microsatellite status, namely MSI-H, MSI-L, and MSS. Fold changes (FCs) of the expression levels for each mRNA, lncRNA, and miRNA were then calculated, with $\mid \mathrm{FCl}>2$ and Padj $<0.05$ indicating statistical significance. Given that MSI-H and MSS COAD had the most differentially expressed RNAs, we then shifted our focus to the MSI-H group and MSS group during the subsequent analysis. To improve the accuracy and credibility of the prediction model, differentially expressed RNAs with $|\mathrm{FC}|>2$ and Padj $<0.01$ were considered statistically significant. The analysis of differentially expressed RNAs was performed using the edge $R$ package in $R$.

\section{Construction of the ceRNA network}

Construction of the ceRNA network was based on the theory that lncRNAs can adsorb miRNAs given their "miRNA sponge" function, which in turn regulates mRNAs. The miRcode (http://www.mircode.org/), starBase (http:// starbase.sysu.edu.cn/), mirTarBase (http://mirtarbase. cuhk.edu.cn/), and spongeScan (http://spongescan.rc.ufl. edu) databases were all used to predict IncRNA-mRNA and miRNA-mRNA interactions based on differentially expressed lncRNAs, miRNAs, and mRNAs according to MSI status. Prediction results were intersected with differentially expressed RNAs to construct a network. The above-mentioned steps were performed in $\mathrm{R}$ using the GDCRNATools package. Cytoscape (version 3.2.1) was used to build the lncRNA-miRNA-mRNA network. The Network Analyzer tools in Cytoscape were then utilized to calculate the degree, after which the top 5 subnetworks were filtered out according to Table Panel.

\section{Functional enrichment analysis}

We used the topGO package in R and KOBAS3.0 (http:// kobas.cbi.pku.edu.cn/kobas3) for enrichment analysis. Fisher's exact test was used to determine the significant levels of enrichment. Biological processes in GO and KEGG were searched for pathways, and the significance level was set at $\mathrm{P}<0.05$.

\section{Survival analysis}

Differentially expressed miRNAs and lncRNAs obtained from TCGA database were used for survival analysis. The univariate Cox proportional hazard model was used to analyze the effects of gene expression level on the overall survival (OS) of patients with COAD. Samples were divided into upper and lower quartiles according to gene expression level, with the upper and lower quartiles having 75 samples for analysis. The 10 top genes in terms of $\mathrm{P}$ values analyzed using the Cox proportional hazard model underwent Kaplan-Meier survival analysis, after which survival curves of both groups were compared using the log rank test, with $\mathrm{P}<0.01$ indicating statistical significance.

\section{Statistical analysis}

The relationship between clinical features and microsatellite status was analyzed using the Chi-square test in $\mathrm{R}$, with $\mathrm{P}<0.05$ indicating statistical significance.

\section{Results}

\section{Microsatellite status and clinical feature analysis}

To explore the relationship between MSI status and basic clinical features, this study analyzed the age, sex, tumor stage, TNM stage, and anatomic neoplasm subdivisions contained in TCGA database (Table 1). The descending colon, sigmoid colon, rectosigmoid junction, and splenic flexure were defined as the left colon, whereas the rest of the regions were defined as the right colon. Our results showed that MSI status was associated with tumor $\mathrm{N}$ stage, tumor stage, and anatomic neoplasm subdivision, but not with sex, age, $T$ stage, and $M$ stage.

\section{Differentially expressed RNAs according to MSI status}

A total of 363 COAD samples from TCGA database were divided into 3 groups according to their MSI status and were subsequently analyzed, after which differentially expressed lncRNAs, miRNAs, and mRNAs were identified 
Table 1 Differences in the frequencies of MSI status by clinicopathological features in colon adenocarcinoma cases in TCGA database

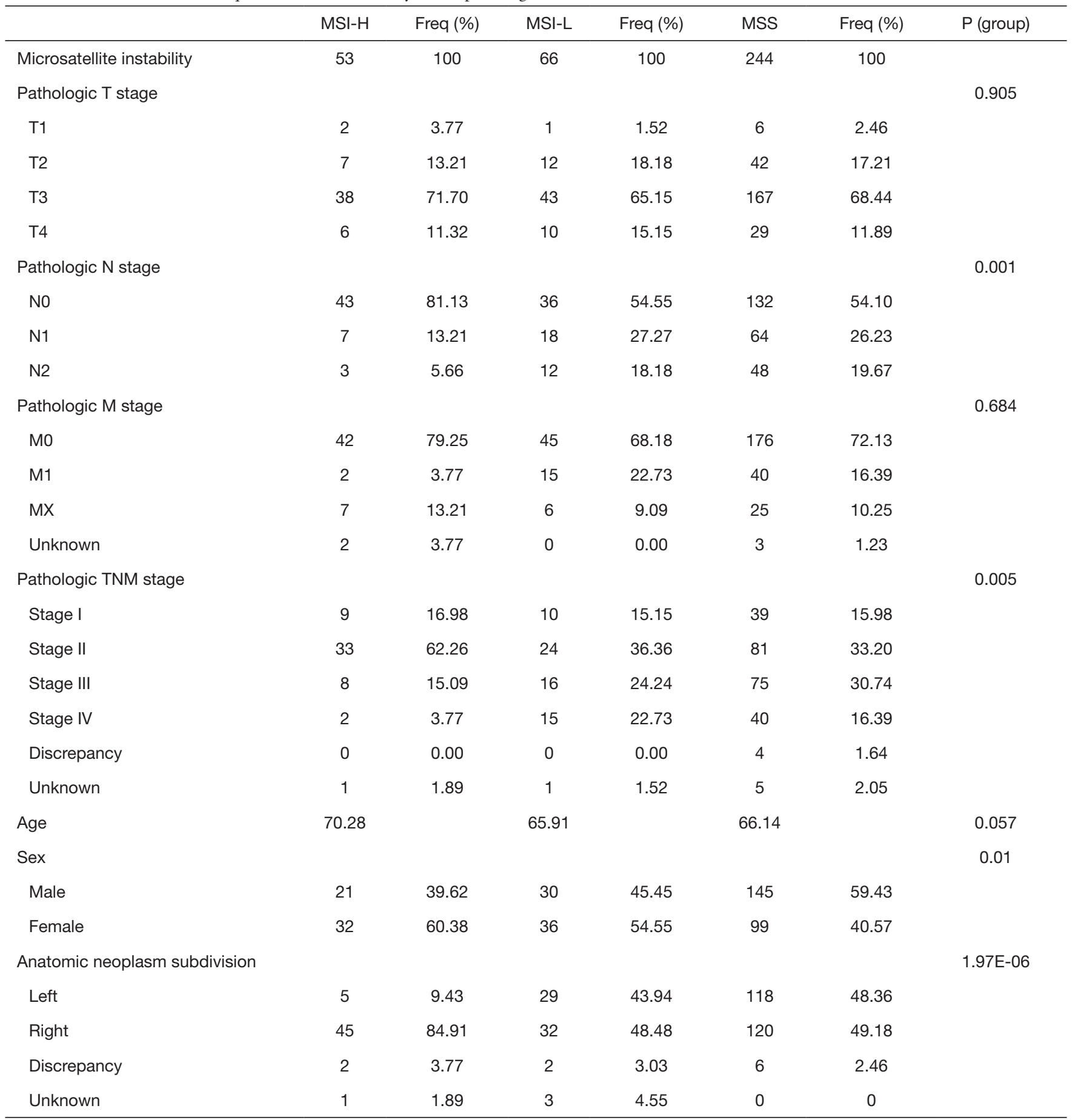

TCGA, The Cancer Genome Atlas; MSS, microsatellite stable; MSI-L, microsatellite instability low; MSI-H, microsatellite instability high; left colon, descending colon, sigmoid colon, rectosigmoid junction, and splenic flexure; right colon, the rest of the regions were defined as the right colon. 

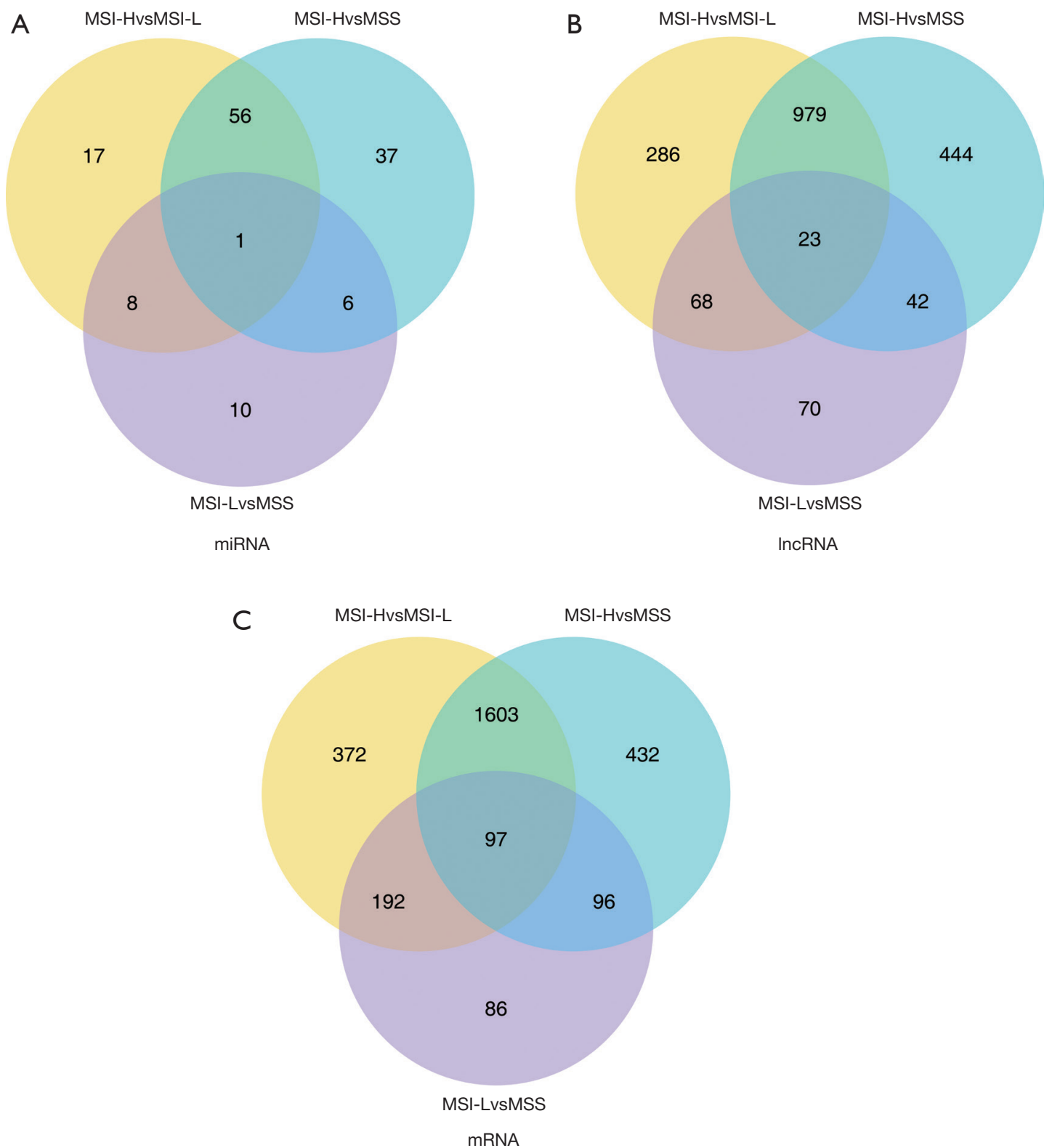

Figure 1 Venn diagram analysis of differentially expressed miRNAs, lncRNAs, and mRNAs between different microsatellite instability statuses using a broad standard ( $\mid \mathrm{FCl}>2, \mathrm{Padj}<0.05)$. (A) Venn diagram analysis of miRNAs; (B) venn diagram analysis of lncRNAs; (C) venn diagram analysis of mRNAs.

( $\mid \mathrm{FCl}>2$, Padj <0.05) (Figure 1). A total of 82, 1,280, and 2,121 differentially expressed miRNAs, IncRNAs, and mRNAs were found in the MSI-H (53 samples) and MSS (244 samples) groups, respectively, after adopting more stringent analysis standards $(\mid \mathrm{FCl}>2, \mathrm{Padj}<0.01)$ (Figure 2). Available online: https://cdn.amegroups.cn/static/public/ tcr-21-2380-1.pdf lists the top 25 RNAs for upregulated and downregulated genes.

\section{Prediction of miRNA targets and construction of the ceRNA network}

The ceRNA network was constructed based on miRNAs. The database was used to predict the targets of the differentially expressed miRNAs while simultaneously determining whether lncRNAs/mRNAs significantly share miRNAs and whether the similarity of the targeted 
A MSI-HvsMSS

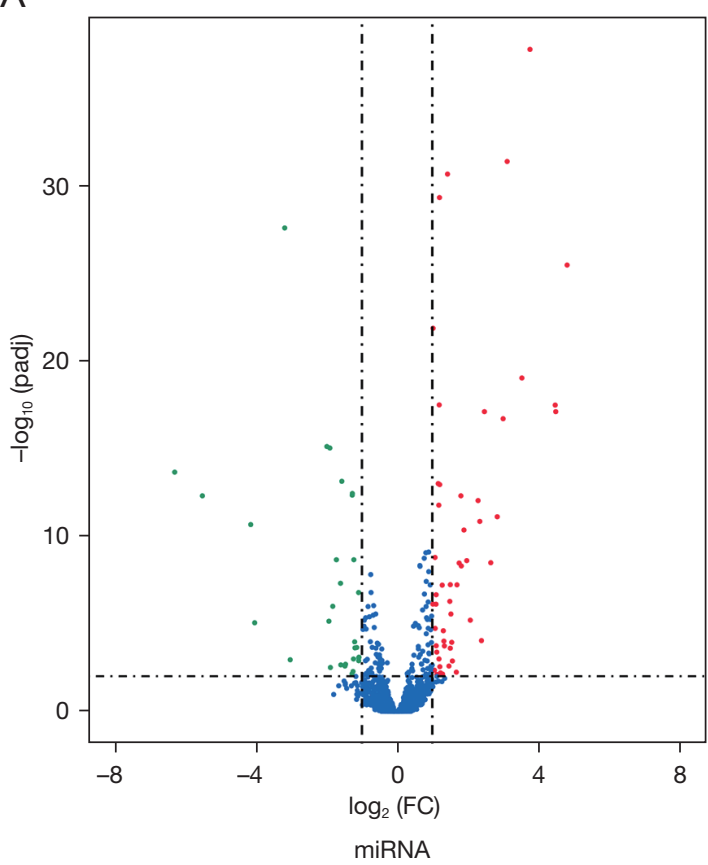

C
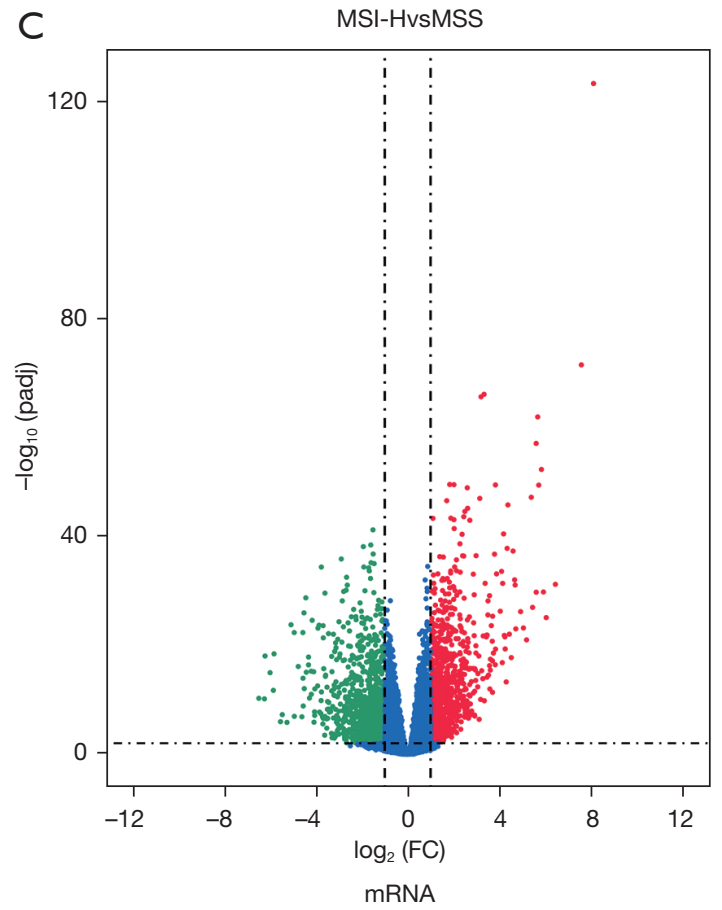

B

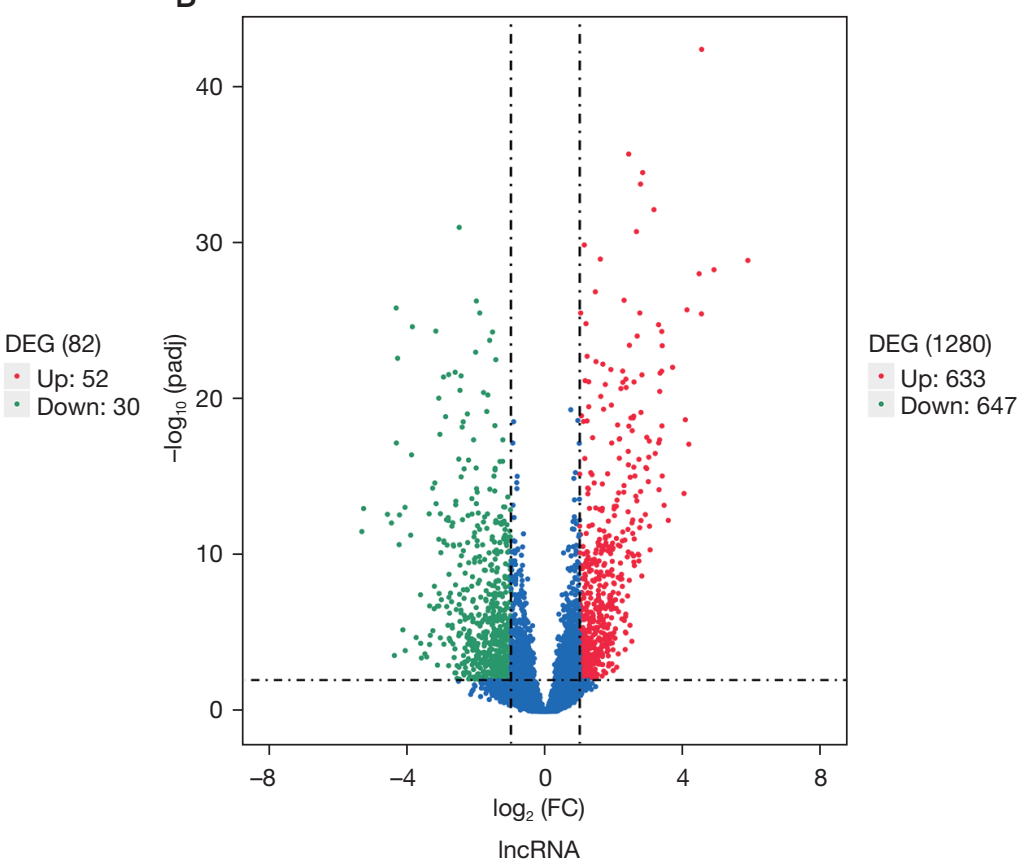

DEG (2121)

$$
\text { - Up: } 1011
$$

- Down: 1110

Figure 2 Volcano plot diagram analysis of differenyially expressed miRNAs, lncRNAs, and mRNAs between MSI-H group and MSS group with a more stringent analysis standards. (A) Volcano plot diagram analysis of miRNAs; (B) volcano plot diagram analysis of lncRNAs; (C) volcano plot diagram analysis of mRNAs. 


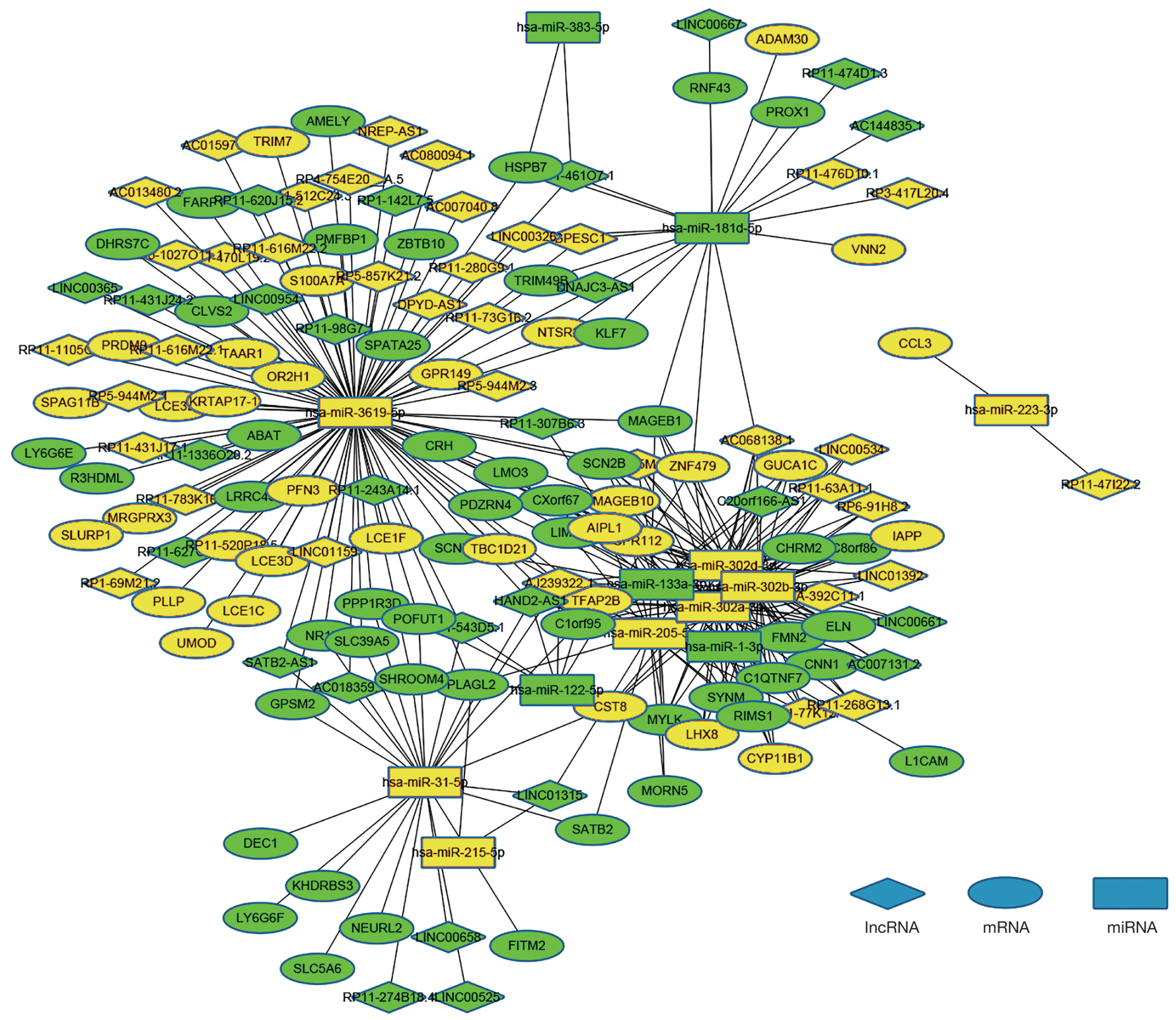

Figure 3 The lncRNA-miRNA-mRNA competing endogenous RNA network. The rectangles indicate miRNAs, ellipses represent mRNAs, and diamonds represent lncRNAs. Yellow nodes indicate upregulated RNAs while green nodes indicate downregulated RNAs.

regulation mode connects lncRNAs and mRNAs, thereby increasing the accuracy of the ceRNA network. The predicted results were compared with the differentially expressed genes, selecting those that appeared in both groups as network nodes to build the network. Following differential expression analysis, 82 miRNAs, 1,280 lncRNAs, and 2,121 mRNAs were identified. The following principles were used to increase the accuracy of the ceRNA network: (I) a hypergeometric test was utilized to determine whether
lncRNAs/mRNAs shared miRNAs (Pearson correlation coefficient $\mathrm{P}<0.05$ ); (II) miRNAs negatively regulated lncRNAs/mRNAs, while lncRNA and mRNA expression was positively correlated; (III) miRNA-lncRNA/miRNAmRNA was similarly regulated, with similarity score not equaling 0; (IV) screening sensitivity correlation and Pearson correlation coefficient were utilized (11) (Figure 3).

After analyzing the network using Network Analysis in Cytoscape, a subnetwork based on the top 5 miRNAs 


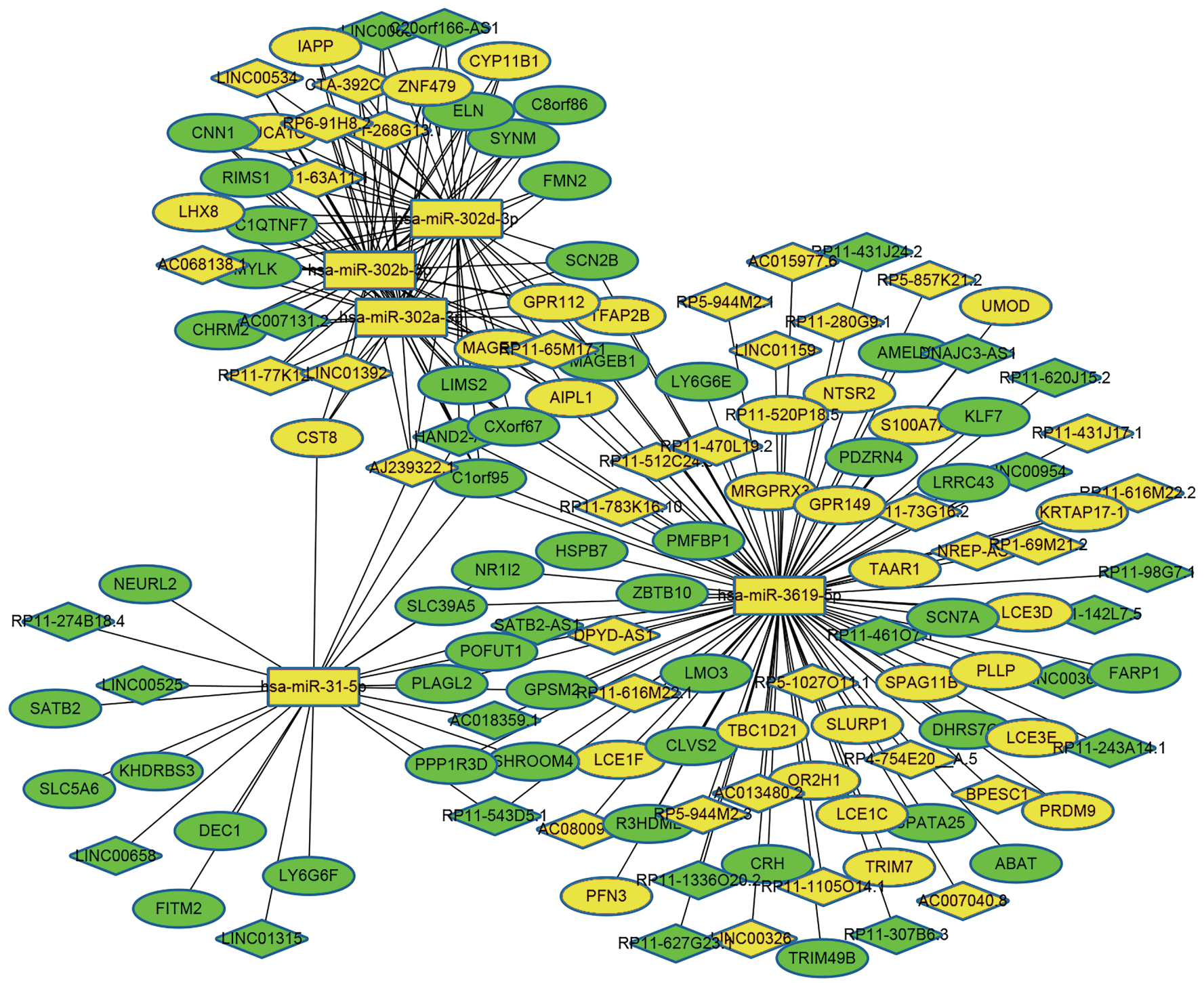

Figure 4 The subnetwork of the lncRNA-miRNA-mRNA competing endogenous RNA network based on the top 5 miRNAs. The rectangles indicate miRNAs, ellipses represent mRNAs, and diamonds represent lncRNAs. Yellow nodes indicate upregulated RNAs while green nodes indicate downregulated RNAs.

was created. As shown in Figure 4, hsa-miR-302a-3p, hsamiR-302b-3p, hsa-mir-302d-3p, hsa-miR-31-5p, and hsamiR-3619-5p together constituted the cornerstone of the subnetwork. The subnetworks corresponding to each miRNA are shown in Figure 5.

\section{GO and KEGG pathway analysis of differentially expressed genes}

To further explore the role of differentially expressed RNAs in tumorigenesis and development, GO function analysis and KEGG pathway enrichment analysis were conducted. Differentially expressed mRNAs/lncRNAs were divided into 2 groups (upregulated and downregulated) for analysis in R. The top 20 GO biological processes for upregulated and downregulated genes were selected based on $\mathrm{p}$ values, as shown in Figure 6. The top 20 KEGG pathways for the upregulated and downregulated mRNAs/lncRNAs are shown in Figure 7. Among these pathways, cytokine-cytokine receptor interaction, natural killer (NK) cell-mediated cytotoxicity, inflammatory bowel disease, intestinal immune network for IgA production, and cell adhesion molecules 

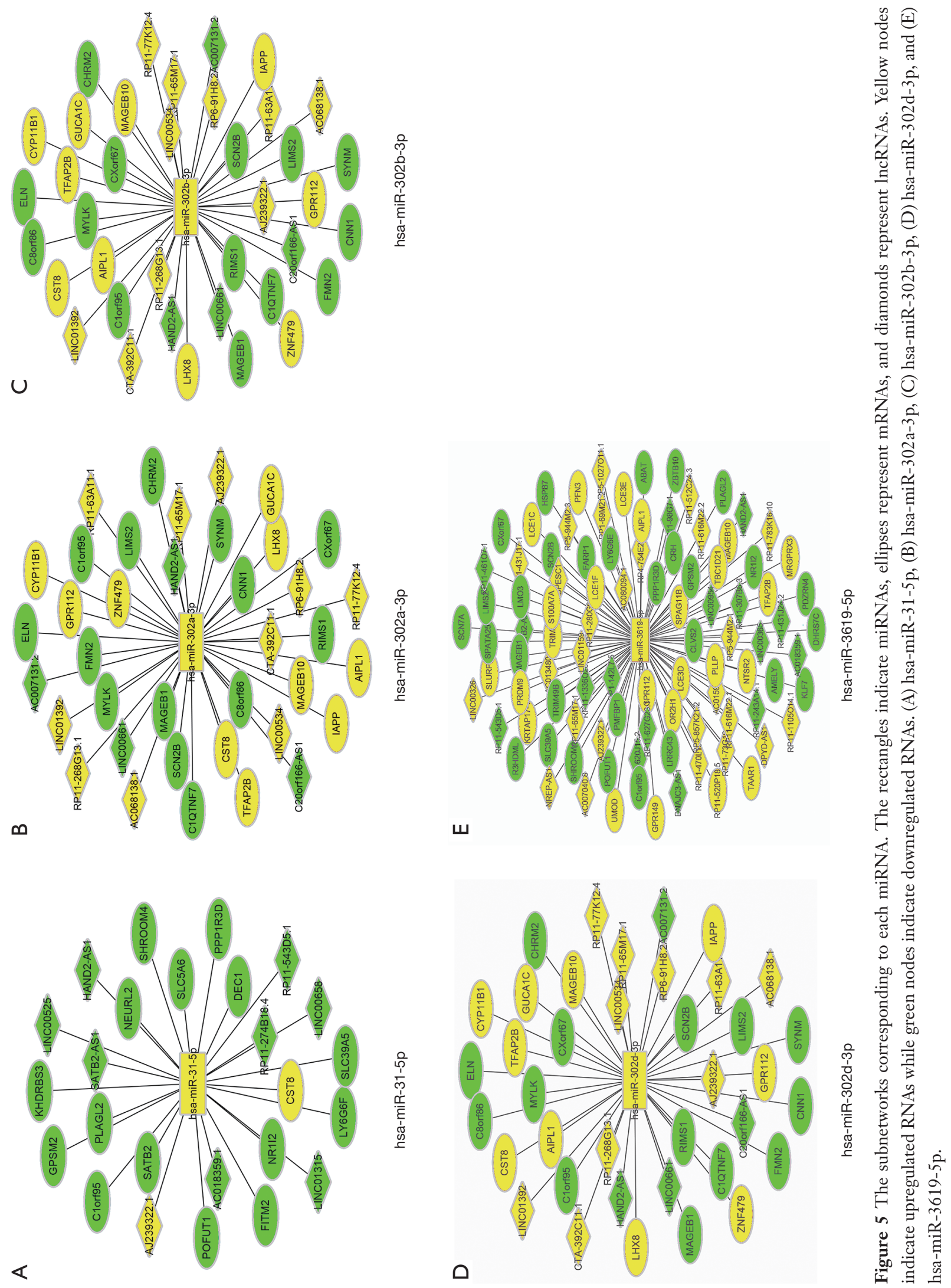


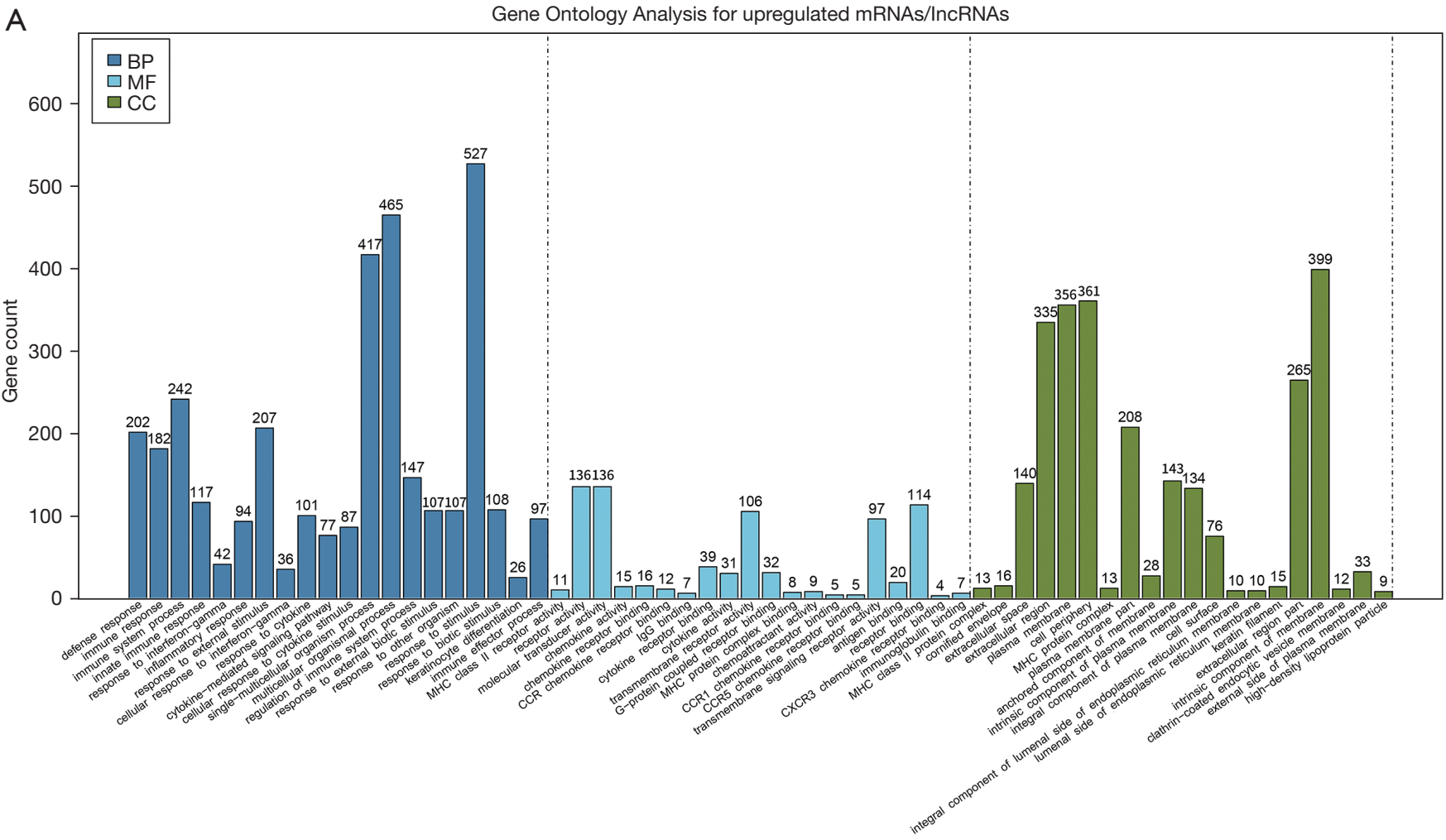

B

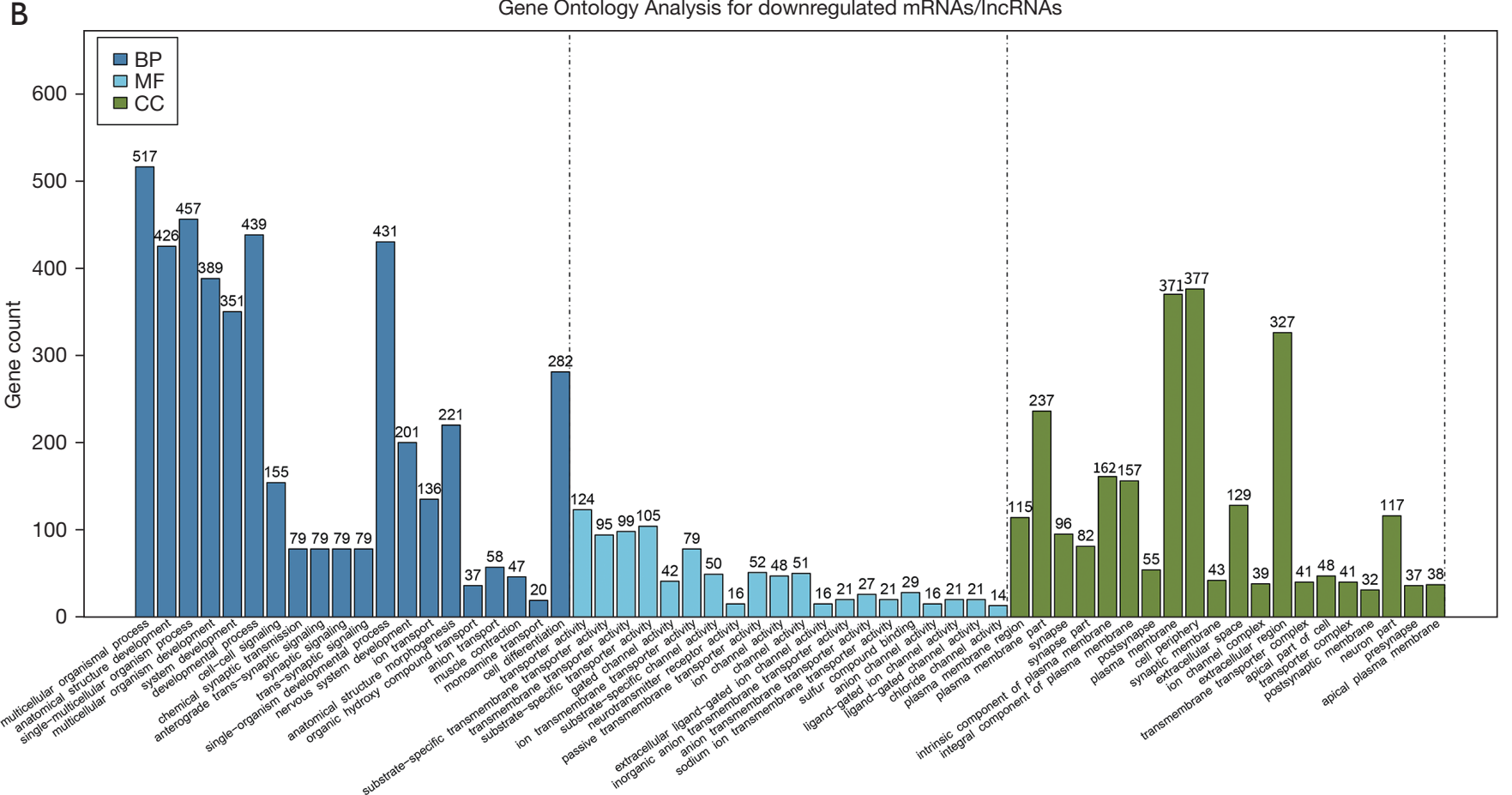

Figure 6 Top 20 Gene Ontology (GO) analysis results of upregulated and downregulated mRNAs/lncRNAs, respectively. Dark blue bars indicate Biological Process (BP), light blue bars indicate Molecular Function (MF), and green bars indicate Cellular Component (CC). (A) Top 20 Gene Ontology (GO) analysis results of upregulated mRNAs/lncRNAs. (B) Top 20 Gene Ontology (GO) analysis results of downregulated mRNAs/lncRNAs. 


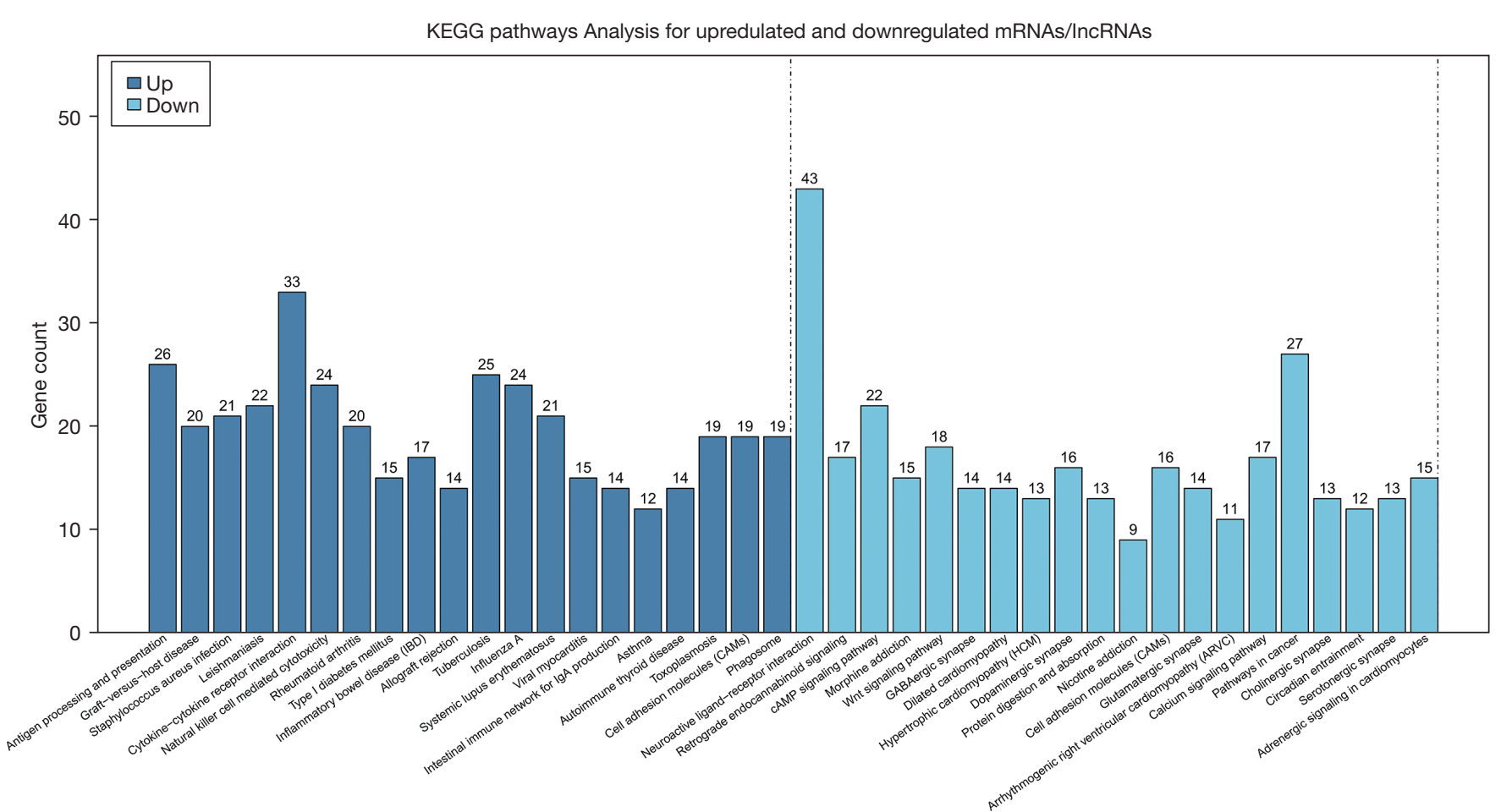

Figure 7 Top 20 Kyoto Encyclopedia of Genes and Genomes (KEGG) analysis results of upregulated and downregulated mRNAs/ lncRNAs, respectively. Dark blues bars indicate results of upregulated mRNAs/lncRNAs, light blue bars indicate results of downregulated mRNAs/lncRNAs.

were upregulated, whereas the cAMP signaling pathway, Wnt signaling pathway, and calcium signaling pathway were downregulated. These pathways have been reported to be associated with tumorigenesis and development and may play an important role in cancer treatment (12-19). GO analysis results for these genes mainly highlighted the functions that need to be suppressed in the process of tumorigenesis and development, such as neurotransmitters, cell membrane receptors, and immune responses.

Given that the differentially expressed miRNAs targeted lncRNAs and mRNAs, GO and KEGG analyses of miRNAs were based on the target lncRNAs and mRNAs in the network (Figure 8). These results all indicated that miRNAs play an important role in the occurrence and development of tumors, particularly in terms of cell function and pathway function.

\section{Survival analysis}

Kaplan-Meier survival analysis aided in further understanding the clinical value of differentially expressed miRNAs and lncRNAs. Given that univariate Cox analysis showed no significant results for miRNAs, we relaxed the cutoff for miRNAs to $\mathrm{P}<0.1$, after which 7 miRNAs were ultimately identified and used for survival analysis. Unfortunately, these miRNAs showed no significant effects on survival (Figure 9). On the other hand, 10 lncRNAs (Figure 10) were found to be significantly associated with prognosis. As demonstrated by the Kaplan-Meier survival curves shown in Figure 10, with the increase of expression level, AC005235.1, LINC01485, PTPRD-AS1, RP1102D24.5, RP5-119A7.14, RP11-429J17.8, RP11-543D5.1, and RP11-86516.2 were positively associated with OS (log rank $\mathrm{P}<0.05)$, with $\mathrm{AC} 003986.7$ and $\mathrm{AC} 012531.25$ having the worst OS $(\log$ rank $\mathrm{P}<0.05)$.

\section{Discussion}

CRC is the fourth most common and second most fatal cancer worldwide (1), with COAD being the most common pathological type of CRC. Following the largescale application of immunotherapy, drastic improvements in the prognosis and quality of life of patients with CRC have been noted. The success of the KEYNOTE-177 
A

Gene Ontology Analysis of differentially expressed miRNAs

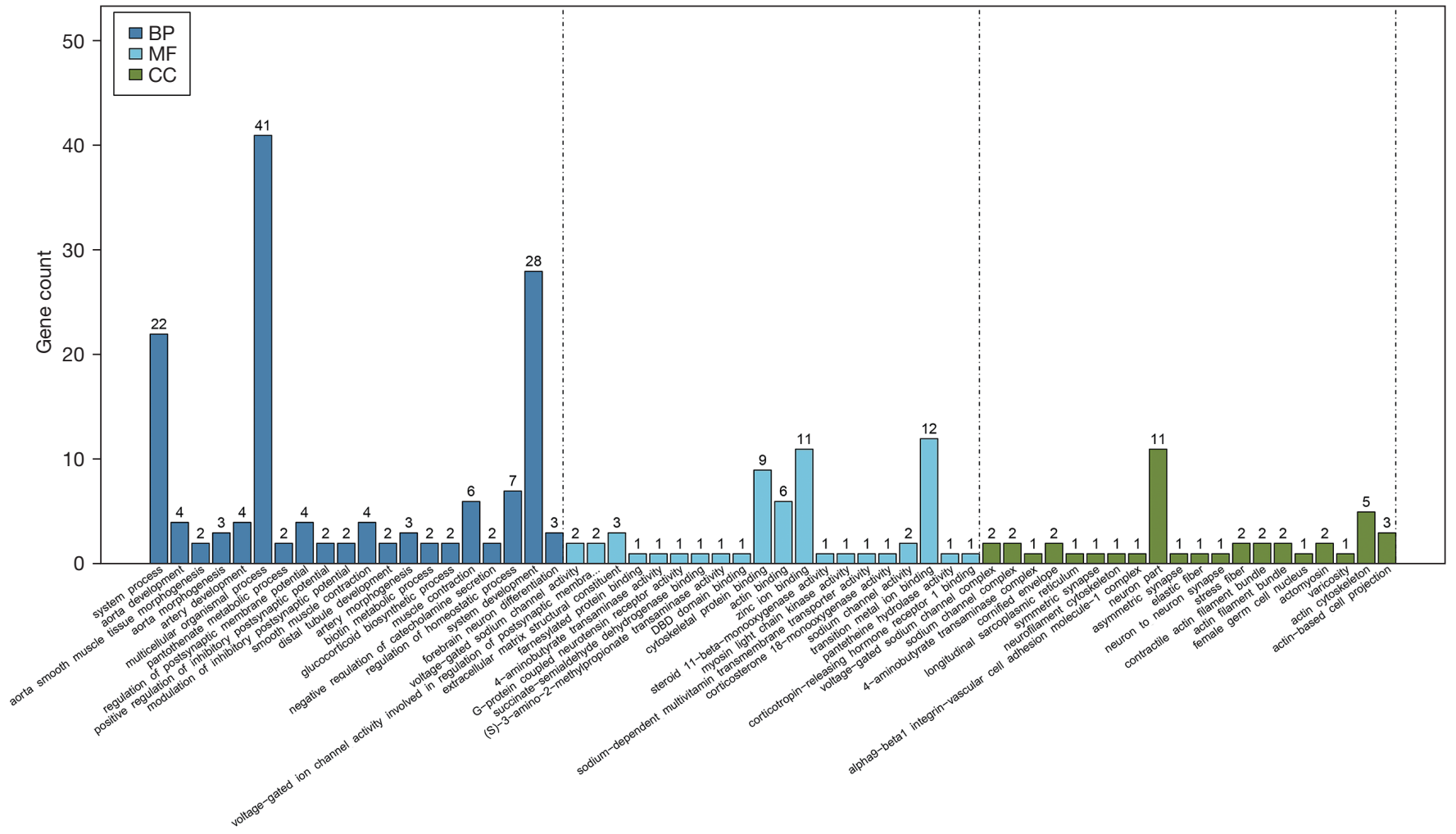

B

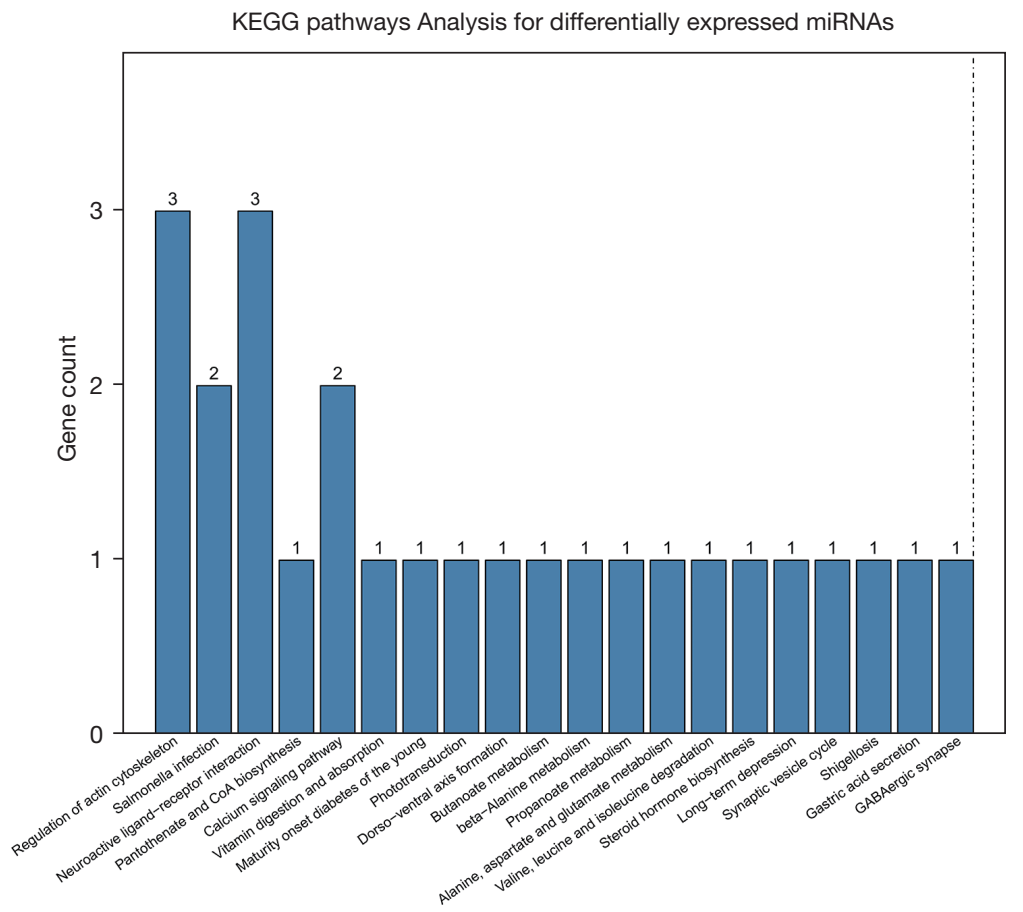

Figure 8 Gene Ontology (GO) and Kyoto Encyclopedia of Genes and Genomes (KEGG) analyses results of differentially expressed miRNAs. (A) GO analyses results. Dark blue bars indicate Biological Process (BP), light blue bars indicate Molecular Function (MF), and green bars indicate Cellular Component (CC). (B) KEGG analyses results. 

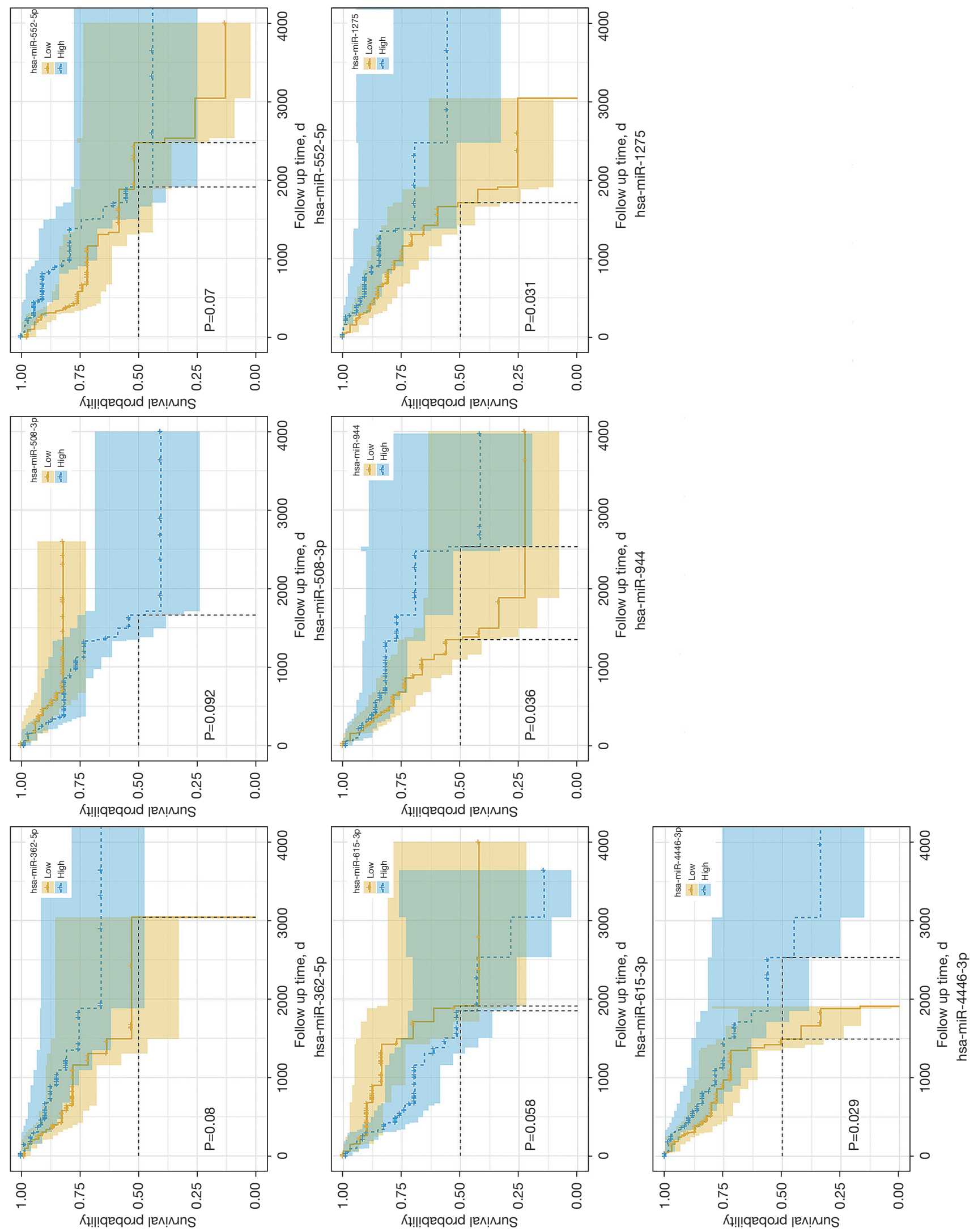

总 

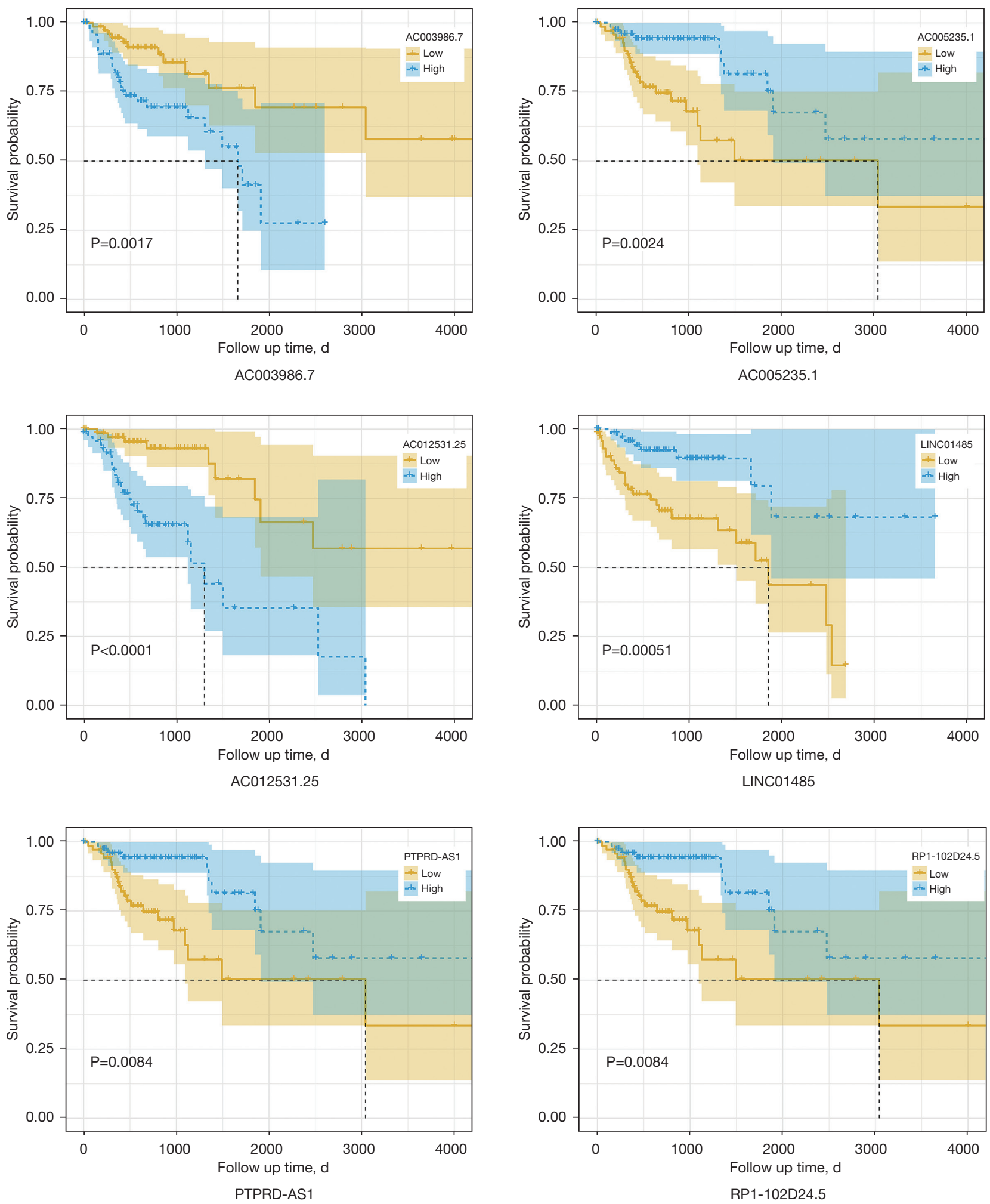

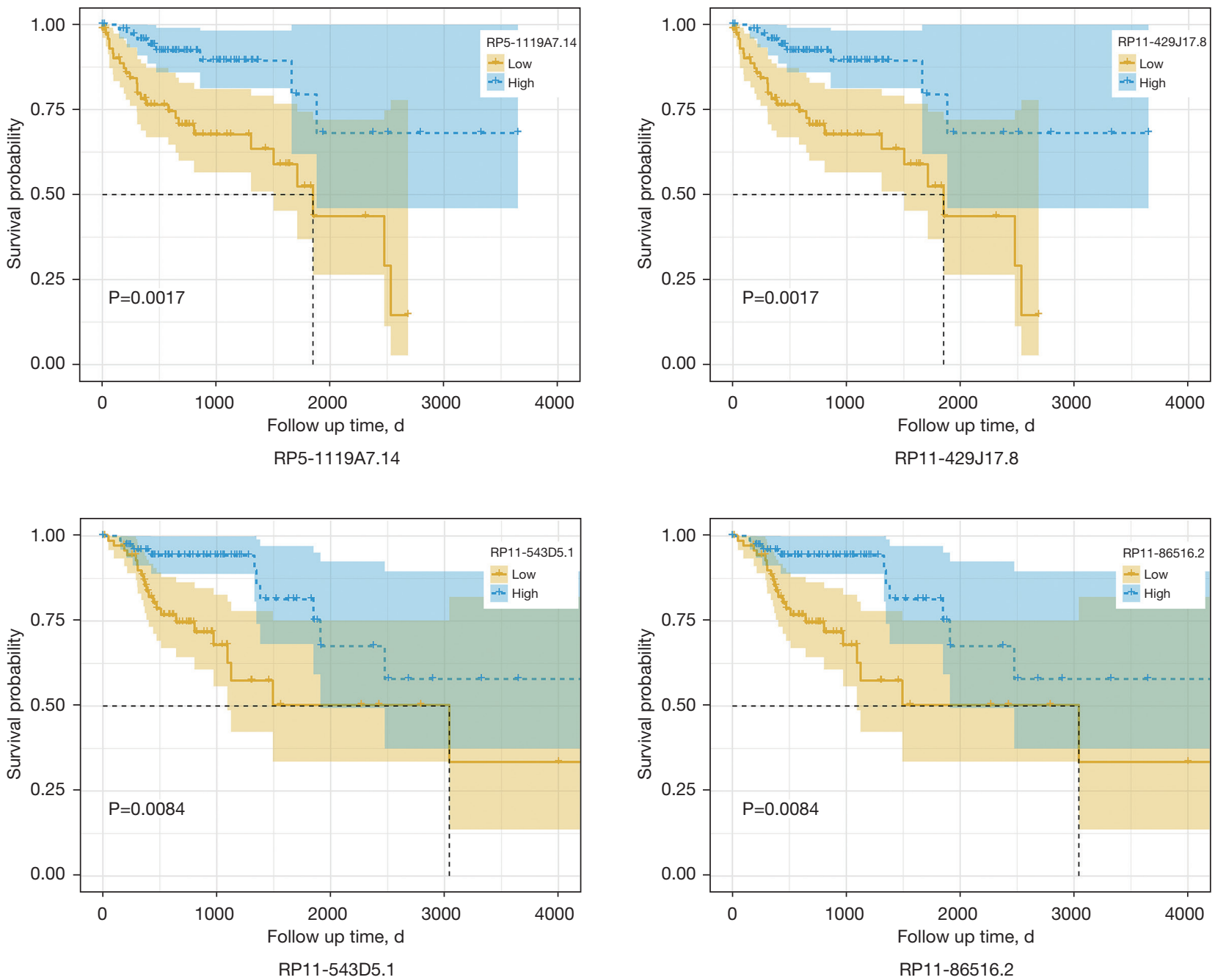

Figure 10 Kaplan-Meier survival curves for 10 differentially expressed lncRNAs. Horizontal axis, overall survival time, years; vertical axis, survival function.

trial prompted the FDA to approve immunotherapy as a first-line treatment for CRC (5). However, given that immunotherapy is only effective for patients with MSI-H/ pMMR CRC, not all patients may benefit from the new generation of cancer therapy (7). As such, uncovering the mechanism for the development of MSI can be a key step in expanding the scope of immunotherapy. Our goal is to analyze different microsatellite status based on the ceRNA theory, in order to provide researchers with new ideas, and work together to expand the scope of immunotherapy in patients with colorectal cancer, so that more patients can learn from Benefit from immunotherapy.

Following Salmena et al.'s proposal of the ceRNA network hypothesis, the relationship between miRNAs and lncRNAs and tumor progression has become more complicated (8). While recent studies have shown that lncRNAs and miRNAs can form a control axis that regulates the development of cancer $(20,21)$, very few publications have focused on the ceRNA network in CRC with MSI. As such, the current study aimed to determine the mechanism for the development of different MSI types under the ceRNA hypothesis and provide novel concepts for enhancing the efficacy of immunotherapy.

The present study identified differentially expressed mRNAs, IncRNAs, and miRNAs according to MSI status, after which their expression profiles were compared between the MSI-H and MSS groups. GO and KEGG analyses allowed us to further determine the functions and pathways 
involved in the differential expression of RNAs. We then established a ceRNA network using bioinformatics analysis tools. Prognostic analysis of significantly differentially expressed miRNAs, lncRNAs, and mRNAs was conducted to explore their potential as novel targets for enhancing immunotherapy and prognostic biomarkers.

Based on the regulation model of RNAs in the ceRNA hypothesis, a regulatory network of differentially expressed genes was constructed using bioinformatics tools. After selecting the degree of each node, a subnetwork based on 5 miRNAs was established, through which the interactions between miRNAs, lncRNAs, and mRNAs were described to better explain the COAD MSI. Among them, 5 cornerstone miRNAs have been confirmed to play vital roles in tumorigenesis, development, and drug action. Previous experiments have shown that the absence of miR-31 in mammary stem cells impairs the growth of tumor cells and decreases tumor-initiating ability and metastasis (22). In CRC, the combination of FOXC1 and hsa-miR31-5p and the increase in miR-31-5p have been among the reasons for oxaliplatin resistance (23). One report found that miR-31, which is highly expressed in patients with colitis, protected mouse intestinal epithelium by inhibiting the expression of inflammatory cytokine receptors (IL7R and IL17RA) and signal proteins (GP130) (24). MiR-302a, miR-302b, and miR-302d are all important members of the miR-302 family that play a regulatory role in embryonic stem cell renewal and the maintenance of pluripotency (25). Recent studies have suggested that they also participate in tumorigenesis, immunity, and other diseases. MiR-302 blocks the cell cycle transition from the $\mathrm{G} 1$ to $\mathrm{S}$ phase by inhibiting the cyclin E-CDK2 and cyclin D-CDK4/6 pathways, thereby inhibiting the tumorigenicity of human pluripotent stem cells (25). Meanwhile, Guo et al. found that miR-302 overexpression had the same effect as IGF-1R silencing such that both could reduce AKT phosphorylation to regulate cell cycle-related proteins and apoptosis proteins Bax/Bcl2 in gastric cancer (26). This mechanism explains how 5 -fluorouracil reinforces CRC cell death (27). Another study with a novel research concept suggested that miR-302 and GCNT3 can both regulate the ERK pathway and epithelialmesenchymal transition and could potentially be cancer treatment targets (28). Several basic studies have verified that mir-3619, a relatively newly discovered miRNA, can play a regulatory role in the occurrence and development of tumors through the regulatory axis proposed based on the ceRNA hypothesis (29-31). Nonetheless, the discovery of other modes of action of mir-3619 requires more effort.
Based on our analysis and previous experimental results, we believe that these 5 cornerstone miRNAs have the potential to become therapeutic targets to Change the status that MSS COAD is not sensitive to immunotherapy.

The current study utilized GO and KEGG analyses to determine characteristic mRNAs and lncRNAs according to microsatellite status. Based on the ceRNA interaction model hypothesis and the obtained network, we predicted the cellular functions of miRNAs and their roles in various pathways. GO analysis focused on functions that needed to be suppressed for tumorigenesis and development. For KEGG analysis, NK cell-mediated cytotoxicity aroused our interest. NK cells can kill tumor cells through perforin and granzyme and can inhibit tumorigenesis through the ligation of death receptor-mediated pathways. Through their experiments, Hsu et al. found that depletion of NK cells accelerates tumor growth. Accordingly, PD-1-blocking antibody treatment was completely ineffective in a mouse model of NK cell depletion. This proved that NK cells are involved in the therapeutic effect of PD-1/PD-L1 blockade (32). Moreover, Trefny et al. found that NK cells in solid tumors expressed high levels of the immune checkpoint receptor PD-1 on their cell surface and that PD1-positive NK cells expressed more inhibitory receptors. Nonetheless, the impaired tumor infiltrating function of NK cells could be rescued by PD-1 blockade (33). Reports have shown that cancer-associated fibroblasts and tumorassociated macrophages play a synergistic role in the tumor microenvironment and have the ability to regulate CRC NK cells (13), suggesting that NK cells can serve to enhance the effect of immunotherapy.

Through survival analysis, the current study identified novel prognostic biomarkers. Although no miRNAs were identified as prognostic biomarkers, 10 lncRNAs were found to be potentially viable novel biomarkers for survival prediction. Our findings suggest that patients with high expression of AC005235.1, LINC01485, PTPRDAS1, RP1-102D24.5, RP5-119A7.14, RP11-429J17.8, RP11-543D5.1, and RP11-86516.2 would have better survival, whereas those with increased AC003986.7 and AC012531.25 expression would have worse outcomes. Unfortunately, given that basic biological research on these new markers has yet to be conducted by our peers, more of their biological functions have yet to be discovered.

\section{Conclusions}

In conclusion, the current study constructed a ceRNA 
network with specific miRNAs, lncRNAs, and mRNAs according to the microsatellite status of COAD. We discovered 5 miRNAs that can be considered as targets for immunotherapy. Future research should pay more attention to NK cells to enhance the effects of immunotherapy. Our findings also showed that the 10 lncRNAs can be used as potential survival biomarkers to guide clinical precision treatment. To the best of our knowledge, no large-scale expression data analysis for COAD according to microsatellite status has been available. Our analysis provides new insights that could guide research on immunotherapy targets at the genetic level.

\section{Acknowledgments}

Funding: None.

\section{Footnote}

Reporting Checklist: The authors have completed the REMARK reporting checklist. Available at https://dx.doi. org/10.21037/tcr-21-2380

Conflicts of Interest: All authors have completed the ICMJE uniform disclosure form (available at https://dx.doi. org/10.21037/tcr-21-2380). The authors have no conflicts of interest to declare.

Ethical Statement: The authors are accountable for all aspects of the work in ensuring that questions related to the accuracy or integrity of any part of the work are appropriately investigated and resolved. The study was conducted in accordance with the Declaration of Helsinki (as revised in 2013).

Open Access Statement: This is an Open Access article distributed in accordance with the Creative Commons Attribution-NonCommercial-NoDerivs 4.0 International License (CC BY-NC-ND 4.0), which permits the noncommercial replication and distribution of the article with the strict proviso that no changes or edits are made and the original work is properly cited (including links to both the formal publication through the relevant DOI and the license). See: https://creativecommons.org/licenses/by-nc-nd/4.0/.

\section{References}

1. Bray F, Ferlay J, Soerjomataram I, et al. Global cancer statistics 2018: GLOBOCAN estimates of incidence and mortality worldwide for 36 cancers in 185 countries. CA Cancer J Clin 2018;68:394-424.

2. O'Connell MJ, Colangelo LH, Beart RW, et al. Capecitabine and oxaliplatin in the preoperative multimodality treatment of rectal cancer: surgical end points from National Surgical Adjuvant Breast and Bowel Project trial R-04. J Clin Oncol 2014;32:1927-34.

3. Leung M, Gholami S. The state of hepatic artery infusion chemotherapy in the management of metastatic colorectal cancer to the liver. Chin Clin Oncol 2019;8:54.

4. Heinemann V, von Weikersthal LF, Decker T, et al. FOLFIRI plus cetuximab versus FOLFIRI plus bevacizumab as first-line treatment for patients with metastatic colorectal cancer (FIRE-3): a randomised, open-label, phase 3 trial. Lancet Oncol 2014;15:1065-75.

5. Le DT, Kim TW, Van Cutsem E, et al. Phase II OpenLabel Study of Pembrolizumab in Treatment-Refractory, Microsatellite Instability-High/Mismatch Repair-Deficient Metastatic Colorectal Cancer: KEYNOTE-164. J Clin Oncol 2020;38:11-9.

6. Yamamoto H, Imai K. An updated review of microsatellite instability in the era of next-generation sequencing and precision medicine. Semin Oncol 2019;46:261-70.

7. Le DT, Durham JN, Smith KN, et al. Mismatch repair deficiency predicts response of solid tumors to PD-1 blockade. Science 2017;357:409-13.

8. Salmena L, Poliseno L, Tay Y, et al. A ceRNA hypothesis: the Rosetta Stone of a hidden RNA language? Cell 2011;146:353-8.

9. Zhu X, Bu F, Tan T, et al. Long noncoding RNA RP11757G1.5 sponges miR-139-5p and upregulates YAP1 thereby promoting the proliferation and liver, spleen metastasis of colorectal cancer. J Exp Clin Cancer Res 2020;39:207.

10. Chang $Z$, Huang R, Fu W, et al. The Construction and Analysis of ceRNA Network and Patterns of Immune Infiltration in Colon Adenocarcinoma Metastasis. Front Cell Dev Biol 2020;8:688.

11. Paci P, Colombo T, Farina L. Computational analysis identifies a sponge interaction network between long non-coding RNAs and messenger RNAs in human breast cancer. BMC Syst Biol 2014;8:83.

12. Buhrmann C, Yazdi M, Popper B, et al. Evidence that TNF- $\beta$ induces proliferation in colorectal cancer cells and resveratrol can down-modulate it. Exp Biol Med (Maywood) 2019;244:1-12.

13. Zhang R, Qi F, Zhao F, et al. Cancer-associated fibroblasts enhance tumor-associated macrophages enrichment and 
suppress NK cells function in colorectal cancer. Cell Death Dis 2019;10:273.

14. Arthur JC, Perez-Chanona E, Mühlbauer M, et al. Intestinal inflammation targets cancer-inducing activity of the microbiota. Science 2012;338:120-3.

15. Saadatmand S, de Kruijf EM, Sajet A, et al. Expression of cell adhesion molecules and prognosis in breast cancer. $\mathrm{Br}$ J Surg 2013;100:252-60.

16. Ma R, Zhai X, Zhu X, et al. LINC01585 functions as a regulator of gene expression by the CAMP/CREB signaling pathway in breast cancer. Gene 2019;684:139-48.

17. Park JY, Juhnn YS. cAMP signaling increases histone deacetylase 8 expression via the Epac2-Rap1A-Akt pathway in H1299 lung cancer cells. Exp Mol Med 2017;49:e297.

18. Quandt J, Arnovitz S, Haghi L, et al. Wnt- $\beta$-catenin activation epigenetically reprograms Treg cells in inflammatory bowel disease and dysplastic progression. Nat Immunol 2021;22:471-84.

19. Ibrahim S, Chaigne J, Dakik H, et al. SK4 oncochannels regulate calcium entry and promote cell migration in KRAS-mutated colorectal cancer. Cell Calcium 2021;96:102384.

20. Wang H, Huang C, Yao X. The functions of long noncoding RNAs in colorectal cancer. Transl Cancer Res 2019;8:2192-204.

21. Yang L, Ma J, Li L, et al. Screening key lncRNAs and mRNAs for left-sided and right-sided colon adenocarcinoma based on lncRNA-mRNA functional synergistic network. Transl Cancer Res 2020;9:2312-25.

22. Lv C, Li F, Li X, et al. MiR-31 promotes mammary stem cell expansion and breast tumorigenesis by suppressing Wnt signaling antagonists. Nat Commun 2017;8:1036.

23. Hsu HH, Kuo WW, Shih HN, et al. FOXC1 Regulation of miR-31-5p Confers Oxaliplatin Resistance by Targeting LATS2 in Colorectal Cancer. Cancers (Basel) 2019;11:1576.

24. Tian Y, Xu J, Li Y, et al. MicroRNA-31 Reduces Inflammatory Signaling and Promotes Regeneration in Colon Epithelium, and Delivery of Mimics in

Cite this article as: Li C, Zhu Z, Hou Q, Wang B, Zou L, Liu L, Gong W, Guo H. Revealing potential immunotherapy targets through analysis of a ceRNA network in human colon adenocarcinoma. Transl Cancer Res 2021;10(12):5319-5336. doi: $10.21037 /$ tcr-21-2380
Microspheres Reduces Colitis in Mice. Gastroenterology 2019;156:2281-2296.e6.

25. Lin SL, Chang DC, Ying SY, et al. MicroRNA miR-302 inhibits the tumorigenecity of human pluripotent stem cells by coordinate suppression of the CDK2 and CDK4/6 cell cycle pathways. Cancer Res 2010;70:9473-82.

26. Guo B, Zhao Z, Wang Z, et al. MicroRNA-302b-3p Suppresses Cell Proliferation Through AKT Pathway by Targeting IGF-1R in Human Gastric Cancer. Cell Physiol Biochem 2017;42:1701-11.

27. Liu N, Li J, Zhao Z, et al. MicroRNA-302a enhances 5-fluorouracil-induced cell death in human colon cancer cells. Oncol Rep 2017;37:631-9.

28. Li Q, Ran P, Zhang X, et al. Downregulation of N-Acetylglucosaminyltransferase GCNT3 by miR-302b3p Decreases Non-Small Cell Lung Cancer (NSCLC) Cell Proliferation, Migration and Invasion. Cell Physiol Biochem 2018;50:987-1004.

29. Wu H, Liu B, Chen Z, et al. MSC-induced lncRNA HCP5 drove fatty acid oxidation through miR-3619-5p/AMPK/ PGC1 $\alpha /$ CEBPB axis to promote stemness and chemoresistance of gastric cancer. Cell Death Dis 2020;11:233.

30. Chen X, Zhao S, Li Q, et al. LncRNA NEAT1 Knockdown Inhibits Retinoblastoma Progression by miR3619-5p/LASP1 Axis. Front Genet 2020;11:574145.

31. Liu Z, Mei L, He Z. Long non-coding RNA00882 contributes to platelet-derived growth factor-induced proliferation of human fetal airway smooth muscle cells by enhancing $W n t / \beta$-catenin signaling via sponging miR3619-5p. Biochem Biophys Res Commun 2019;514:9-15.

32. Hsu J, Hodgins JJ, Marathe M, et al. Contribution of NK cells to immunotherapy mediated by PD-1/PD-L1 blockade. J Clin Invest 2018;128:4654-68.

33. Trefny MP, Kaiser M, Stanczak MA, et al. PD-1+ natural killer cells in human non-small cell lung cancer can be activated by PD-1/PD-L1 blockade. Cancer Immunol Immunother 2020;69:1505-17.

(English Language Editor: C. Betlzar) 